Case Report

\title{
Chronic Liver Disease First Time Diagnosed During Pregnancy: A Case Report
}

\author{
Khanum Sa ${ }^{\mathrm{a}}$, Khanam $\mathrm{K}^{\mathrm{b}}$, Ahmed JU ${ }^{\mathrm{c}}$

\begin{abstract}
Pregnancy in women with chronic liver disease (CLD) or cirrhosis is rare as CLD usually causes infertility. With improved management of CLD, pregnancy in patients with CLD is likely to become more common. Pregnancy in a CLD patient is likely to be associated with increased fetal and maternal mortality \& morbidity. Pregnancy outcome may be influenced by the underlying etiology and extent of liver disease. Here we describe the case of a pregnancy in a young woman with CLD first time detected during pregnancy that ultimately resulted in fetal death, but the mother survived after delivery.
\end{abstract}

Key Words: Chronic liver disease; cirrhosis of liver; pregnancy.

(Birdem Med J 2015; 5(2): 107-110.)

\section{Introduction}

Liver diseases that occur during pregnancy are a challenge for health care providers. Certain liver diseases are uniquely associated with pregnancy like hyperemesis gravidarum, acute fatty liver of pregnancy, intrahepatic cholestasis of pregnancy and hemolysis, elevated liver enzymes and low platelets (HELLP) syndrome. ${ }^{1}$ Pregnancy unrelated liver diseases include acute viral hepatitis and chronic liver disease. Pregnancy is uncommon in women with CLD or advanced cirrhosis. However if a woman with CLD becomes pregnant then there is an increased risk of complications such as bleeding from esophageal varices, liver failure and hepatorenal syndrome. ${ }^{2-5}$ Maternal deaths have been reported in advanced cirrhosis mainly due to variceal bleeding. ${ }^{5}$ Spontaneous abortion and increased risk of premature childbirth or stillbirth have been reported in

a. Major (Dr.) Shakila Khanum, FCPS (Obs \& Gyn), Classified Specialist in Obstetrics \& Gynecology, Combined Military Hospital, Dhaka

b. Colonel (Dr.) Khaleda Khanam, FCPS (Obs \& Gyn), Professor \& Head of the Department of Obstetrics \& Gynecology, Armed Forces Medical College, Dhaka

c. Dr. Jamal Uddin Ahmed, FCPS (Medicine), Assistant Professor of Medicine, BIRDEM General Hospital \& Ibrahim Medical College, Dhaka

Address of correspondence: Major (Dr.) Shakila Khanum, Classified Specialist, Department of Obstetrics \& Gynecology, Combined Military Hospital, Dhaka. E-mail: jmldollar@ gmail.com

Received: January 30, 2015

Accepted: June 30, 2015
$15-20 \%$ of pregnancies in women with cirrhosis. ${ }^{6}$ We are reporting the case of pregnancy outcome of a woman with CLD due to undetermined cause.

\section{Case Report}

A 20-year-old primi presented to Combined Military Hospital (CMH), Ghatail, Tangail at 32 weeks of gestation with less fetal movement for one day along with low grade fever, abdominal pain, headache and leg swelling. She was on regular antenatal check up. Her last antenatal visit was one month back and till then pregnancy period was uneventful. After admission she was found anaemic, BP 160/100 mm Hg, abdomen was distended and tender, fetal heart sound was absent. On investigation random blood glucose was $10.5 \mathrm{mmol} / \mathrm{L}$ \& ultrasound of pregnancy profile showed 32 weeks intrauterine fetal death (IUD). So she was referred to CMH, Dhaka with the diagnosis of 32 weeks pregnancy of IUD with sever pregnancy induced hypertension (PIH) with newly detected gestational diabetes mellitus (GDM).

Patient had no history of hematemesis, melaena or altered mental status. There was no past history of jaundice or blood transfusion. Physical examination revealed pallor, bipedal edema, splenomegaly (upto the level of the umbilicus), ascites, bilateral basal lung crepitations. Size of the uterus could not be detected due to ascites There was no jaundice, clubbing, spider telangiectasia or palmer erythema. There was no clinical evidence of portosystemic encephalopathy. She had no history of alcohol or intravenous drug abuse. 
Investigations at admission revealed hemoglobin of 8.0 $\mathrm{gm} / \mathrm{dl}$, mean corpuscular volume (MCV) $97.9 \mathrm{fL}$, total leukocyte count of $17700 / \mathrm{mm}^{3}$ (85\% polymorphs and $10 \%$ lymphocytes), platelets $160,000 / \mathrm{mm}^{3}$, total billirubin $1.8 \mathrm{mg} / \mathrm{dl}$, serum alanine aminotranspharese (ALT) 19 $\mathrm{U} / \mathrm{L}$, alkaline phosphatase $195 \mathrm{U} / \mathrm{L}$, serum albumin 2.0 $\mathrm{gm} / \mathrm{dl}$ and prothrombin time 13.0 seconds (control 12.0 seconds). Her serum creatinine was $0.8 \mathrm{mg} / \mathrm{dl}$, serum potassium $3.2 \mathrm{mmol} / \mathrm{L}$ (corrected by supplement), fasting blood glucose $7.4 \mathrm{mmol} / \mathrm{L} \& 2$ hour post prandial glucose $10.6 \mathrm{mmol} / \mathrm{L}$ (diagnosed as GDM) and TSH level 4.98, FT4 14.5 (diagnosed as subclinical hypothyroidism).

Abdominal ultrasound revealed a coarse hepatic parenchyma, cholelithiasis, moderate splenomegaly, moderate ascites (Fig.-1) and a dead fetus of 35 weeks. Blood culture and urine culture showed no growth. Her viral markers including HBsAg, Anti-HCV were negative. Patient was treated conservatively with blood transfusion, broad spectrum antibiotics and oral diuretics (spironolactone \& frusemide). Obstetric management was given as induction of labour by application of misoprostol in posterior fornix $200 \mathrm{mg} 6$ hourly, but the response was poor. Meanwhile the patient's condition was deteriorating with increasing edema and dyspnea. Parenteral diuretics were added. Patient had persisting fever with decreasing platelet count (75,000/cmm). D-dimer was negative. CT abdomen was done and showed CLD with splenomegaly, evidence of portal hypertension, cholelithiasis, acsites (Fig.-2). Echocardiogram was normal. Intracervical catheter was introduced and high dose misoprostostol (400mg 4 hourly) was given. A dead fetus was delivered on $5^{\text {th }}$ day of induction. After delivery patients condition gradually improved. Blood parameters including platelet count became normal. Further investigations were done to find out the cause of CLD as per advice of Gastroenterologist. Upper gastrointestinal endoscopy showed grade II esophageal varices (Fig.3). Anti-HBc total, ANA, Anti-Sm antibody, ceruloplasmin, ferritin were all normal. As no definite cause of CLD was found, patient was diagnosed as cryptogenic cirrhosis of liver.

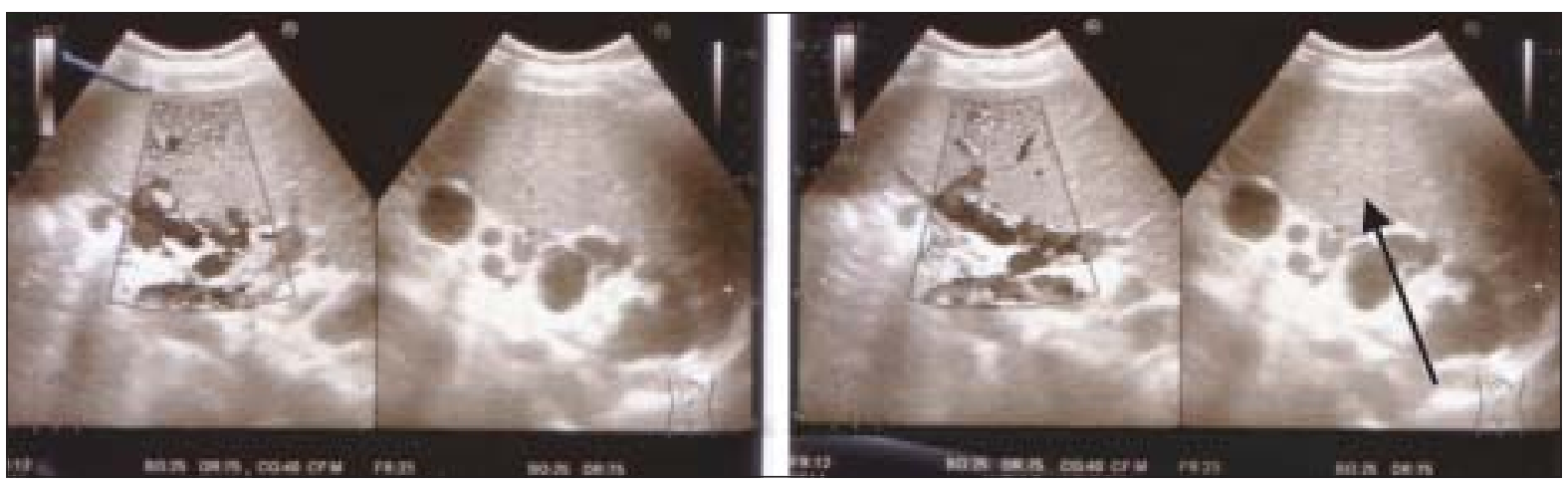

Fig.-1: Abdominal ultrasonography showing splenomegaly (arrow)

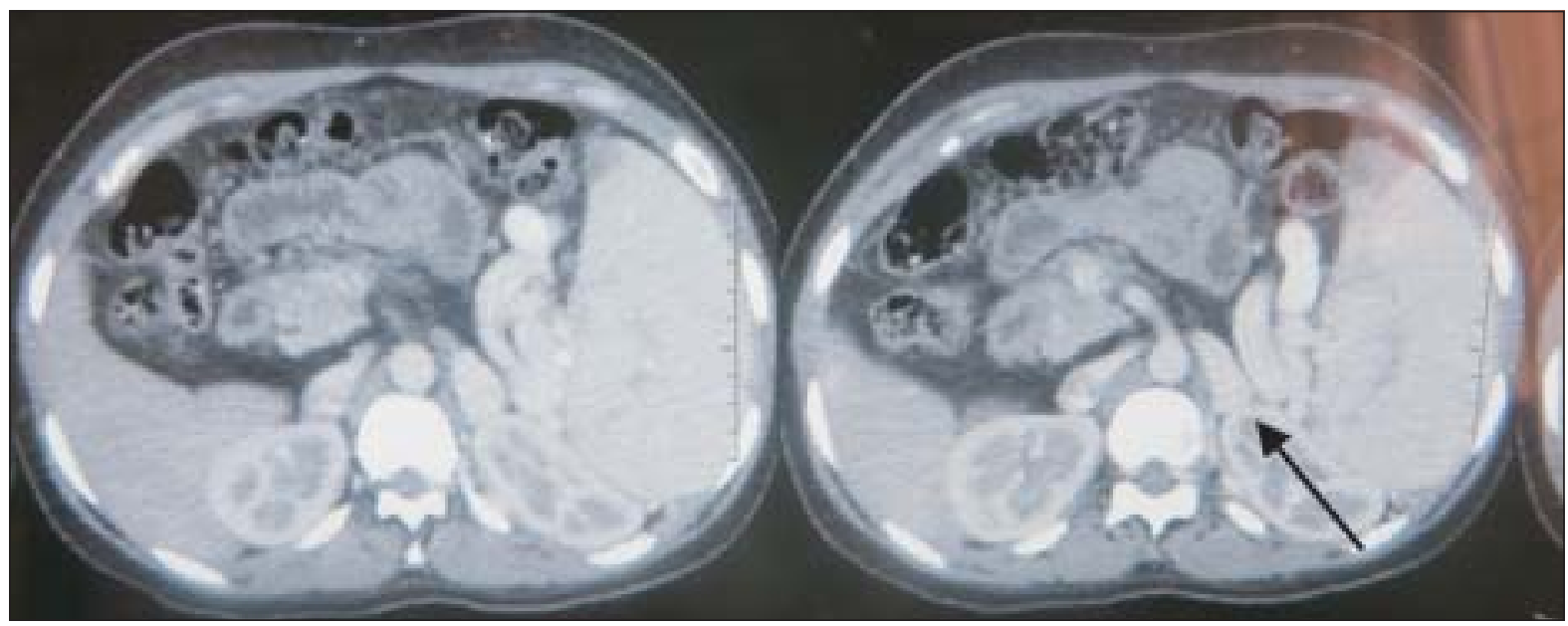

Fig.-2: CT Scan of upper abdomen showing splenomegaly with dilated vessels (arrow) 


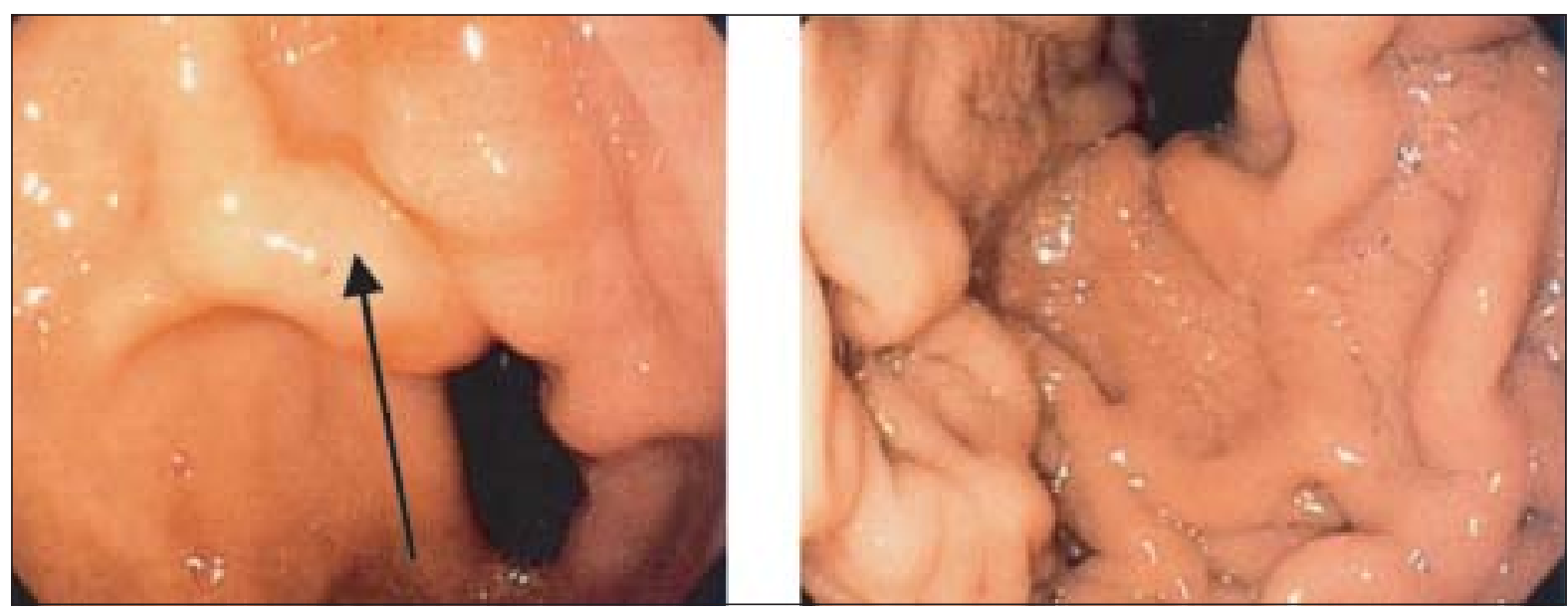

Fig.-3: Endoscopic view of esophageal varices (arrow)

\section{Discussion}

The prevalence of CLD in reproductive-age women approximates 0.45 cases per 1000 . CLD is not a contraindication to pregnancy, but patients with decompensated cirrhosis are unlikely to conceive secondary to hypothalamic pituitary dysfunction. Patients with cirrhosis are often anovulatory and infertility is common even in mild forms of chronic liver diseases. However, women might still become pregnant and should expect a good outcome if their liver function is well compensated (as in non-cirrhotic portal hypertension) and if their liver disease is treated before conception and treatment is maintained during pregnancy. ${ }^{7}$ In our case CLD was first time diagnosed during pregnancy.

Etiology of CLD in pregnancy is similar to that in the non-pregnant state and commonly includes alcohol and viral hepatitis $\mathrm{C}$ and $\mathrm{B}$. Autoimmune hepatitis is also another important cause. The exact cause of CLD in our case could not be determined.

Controversy still remains regarding the effect of pregnancy on cirrhotic patients. Only one study used a control group of non-pregnant cirrhotic patients and found no significant difference in maternal morbidity and mortality, but other studies have demonstrated increased maternal mortality. ${ }^{8-9}$ Advanced CLD increases the risk of maternal and fetal morbidity and mortality. ${ }^{2-3}$ In such cases the stage of the liver disease is the most important determinant of the outcome of the pregnancy. ${ }^{10}$ Patients with cirrhosis and non-cirrhotic portal hypertension are at high risk for premature deliveries. Hepatic decompensation with jaundice, bleeding from esophageal varices, ascites and fulminant liver failure can occur. Our patient presented with ascites and had eosophageal varices. But fortunately she did not develop any variceal bleeding or hepatic failure.

There is an increased rate of spontaneous abortion, premature birth and perinatal deaths in pregnant woman with advanced CLD. Poor fetal prognosis is usually explained by poor condition of the mother in decompensated patients. Though, infants born alive generally remained well. ${ }^{3-4}$ The spontaneous abortion rate in patients with CLD is significantly higher than that of the general population, with a rate of $30 \%$ to $40 \%$ versus $15 \%$ to $20 \%{ }^{11}$ Termination of pregnancy most often occurs as a result of maternal death, variceal hemorrhage, stillbirth, intrauterine growth retardation, and maternal complications during delivery. ${ }^{12}$ In our case the patient presented with intrauterine fetal death.

In a controlled setting vaginal delivery is usually safe and early forceps delivery or vacuum extraction should be considered to prevent any rise in portal pressure due to prolonged straining during labor. ${ }^{3-4}$ Women with cirrhosis generally tolerate laparotomy poorly; therefore the option for caesarean section should be availed with care and caution. Since our patient had IUD with good coagulation profile we opted for vaginal delivery after induction of labour. Although the induction was difficult and prolonged, but it was successful.

Whether to advise pregnancy to a woman with decompensated cirrhosis is a difficult question to answer. However, careful overall assessment of the 
severity of the liver disease as well as of the patient's psychological status and desire for children should lead logically to a resolution of these issues on a case by case basis. With careful monitoring and advanced management, successful pregnancy with a good outcome is a possibility.

\section{References}

1. Tan J, Surti B, Saab S. Pregnancy and cirrhosis. Liver Transpl 2008; 14: 1081-91.

2. Tiribelli C, Rigato I. Liver cirrhosis and pregnancy. Ann Hepatol 2006; 5: 201.

3. Aggarwal N, Sawnhey H, Suril V, Vasishta K, Jha M, Dhiman RK. Pregnancy and cirrhosis of the liver. Aust N Z J Obstet Gynaecol 1999; 39: 503-6.

4. Cerqui AJ, Haran M, Brodribb R. Implications of liver cirrhosis in pregnancy. Aust N Z J Obstet Gynaecol 1998; 38: $93-5$.
5. Zeeman GG, Moise KJ Jr. Prophylactic banding of severe esophageal varices associated with liver cirrhosis in pregnancy. Obstet Gynecol 1999; 94: 842.

6. Lee WM. Pregnancy in patients with chronic liver disease. Gastroenterol Clin North Am 1992; 21: 889-903.

7. Russell MA, Craigo SD. Cirrhosis and portal hypertension in pregnancy. Semin Perinatol 1998; 22: 156-65.

8. Pajor A, Lehoczky D. Pregnancy in liver cirrhosis. Assessment of maternal and fetal risks in eleven patients and review of the management. Gynecol Obstet Invest 1994; 38: 45-50.

9. Borhanmanesh F, Haghighi P. Pregnancy in patients with cirrhosis of the liver. Obstet Gynecol 1970; 36: 315-24.

10. Whelton MJ, Sherlock S. Pregnancy in patients with hepatic cirrhosis: Management and outcome. Lancet 1968; 2: 995-99.

11. Griebel CP, Halvorsen J, Golemon TB, Day AA. Management of spontaneous abortion. Am Fam Physician 2005; 72: 1243-50.

12. Misra S, Sanyal AJ. Pregnancy in a patient with portal hypertension. Clin Liver Dis 1999; 3: 147-62. 